\title{
Room temperature mid-infrared emission from faceted InAsSb multi quantum wells embedded in InAs nanowires
}

\author{
Aiyeshah Alhodaib ${ }^{* 1,2}$, Yasir Noori ${ }^{1}$, Peter J. Carrington ${ }^{3}$, Ana M. Sanchez ${ }^{4}$, Michael D. \\ Thompson $^{1}$, Robert J. Young ${ }^{1}$, Anthony Krier ${ }^{1}$ and Andrew R. J. Marshall*1 \\ ${ }^{1}$ Department of Physics, Lancaster University, Lancaster, LA1 4YB, United Kingdom \\ ${ }^{2}$ Department of Physics, Qassim University, Buryadh 51452, Saudi Arabia \\ ${ }^{3}$ Department of Engineering, Lancaster University, Lancaster, LA1 4YW, United Kingdom \\ ${ }^{4}$ Department of Physics, University of Warwick, Coventry CV4 7AL, United Kingdom
}

Corresponding author e-mails: a.alhodaib@lancaster.ac.uk and a.r.marshall@lancaster.ac.uk 


\begin{abstract}
There is considerable interest in the development of InAsSb based nanowires for infrared photonics due to their high tunability across the infrared spectral range, high mobility and integration with silicon electronics. However, optical emission is currently limited to low temperatures due to strong non-radiative Auger and surface recombination. Here, we present a new structure based on conical type II InAsSb/InAs multi-quantum wells within InAs nanowires which exhibit bright mid-infrared photoluminescence up to room temperature. The nanowires are grown by catalystfree selective area epitaxy on silicon. This unique geometry confines the electron-hole recombination to within the quantum wells which alleviates the problems associated with recombination via surface states, whilst the quantum confinement of carriers increases the radiative recombination rate and suppresses Auger recombination. This demonstration will pave the way for the development of new integrated quantum light sources operating in the technologically important mid-infrared spectral range.
\end{abstract}

\title{
KEYWORDS
}

Nanowires, midwavelength infrared, Photoluminescence, InAsSb multi quantum wells. 
InAsSb nanowires (NWs) have great potential for technologically important applications in nanoscale and quantum devices $^{1,2,3}$. Although most early work has focused on exploiting the transport properties of NWs for electronics ${ }^{4}$, their application to photonics promises many transformational advantages. Catalyst-free selective area epitaxy can be used to produce high quality NWs in precise geometric arrangements, which can be used for light-matter coupling and to tune optical properties ${ }^{5,6}$ as well as facilitating scalable lithographic integration ${ }^{7}$. Whereas infrared photodetectors using these effects have been demonstrated ${ }^{8,9}$, complementary infrared sources are still undeveloped. The principle challenges are in achieving efficient light emission without the requirement for cryogenic cooling, which in turn necessitates incorporating quantum nanostructures into the NW. Here we demonstrate the first room temperature mid-infrared light emission from entirely new faceted, conical InAsSb multi-quantum wells (MQWs) embedded within InAs NWs.

Structural studies of InAs NWs have reported that the wurtzite (WZ) crystal phase dominates and the addition of Sb drives a change to a zinc-blende (ZB) phase ${ }^{10,11}$. While low temperature PL has been observed from InAs NWs up to $130 \mathrm{~K}^{11,12}$, achieving emission at room temperature is vital for realising practical applications. The addition of small amounts of Sb has been shown to suppress lattice defects, including twinning and stacking faults in InAsSb NWs, in agreement with our recent findings (supplementary information fig. S3). However, the PL quenching behaviour is only marginally improved ${ }^{13}$ since the band-edge perturbations introduced by these defects do not strongly influence non-radiative recombination. Recently, in-situ growth of a wider bandgap shell to supress non-radiative surface recombination has been demonstrated using InAsP ${ }^{14}$ on InAs NWs, which enhances emission intensity and enables $\mathrm{PL}$ at room temperature.

In addition to supressing non-radiative recombination, the PL intensity and quenching temperature can be increased by improving the radiative recombination rate. In the present work we target both these enhancements using a type II InAsSb/InAs MQW structure in the NWs, which conveniently enables the emission wavelength to be tuned across the entire mid infrared spectral range. Scanning tunnelling electron microscopy (STEM) studies illustrate the unique faceted conical structural features of these MQWs, while temperature and power dependent PL characterisation evidences the band structure and demonstrates enhanced PL emission up to room temperature. 
Pure InAs NWs, InAsSb NWs and InAs NWs containing InAsSb/InAs MQWs have been grown on silicon (111) substrates, using selective area molecular beam epitaxy (MBE) as described in the methods and shown in fig 1(a). The MQW were formed from 10 repeats of nominally $25 \mathrm{~nm}$ InAs and $8 \mathrm{~nm} \mathrm{InAs}{ }_{1-\mathrm{x}} \mathrm{Sb}_{\mathrm{x}}$ layers, giving a total active region thickness of $330 \mathrm{~nm}$. The MQW NWs had a regular hexagonal cross-section with $\{10 \overline{1} 0\}$ sidewalls, faceted tips and a twinned WZ crystal structure, which is characteristic for InAs NWs ${ }^{15}$. In common with others we have found that the addition of Sb over extended durations, to form bulk InAsSb NWs, drives a stable rotation in the layer stacking, leading to a predominantly ZB structure with a flat wire top. However, in our MQW NWs the InAsSb growth was limited to nanoscale QWs and energy dispersive X-ray spectroscopy (EDXS) mapping revealed preferential incorporation of the Sb on specific crystal planes. This resulted in the formation of a novel QW structure with a faceted, flat-topped, conical shape, as shown in fig. 1(b). Although direct measurement of the Sb fraction in the wells was not possible, InAsSb NWs grown under the conditions used in this work, contained 6-7\% Sb, in good agreement with earlier work that reports a saturation at this concentration ${ }^{16}$. It is noted that in other NW studies, authors have considered nanoscale axial heterostructures as both quantum dots (QDs) ${ }^{17,18}$ and QWs ${ }^{19,20,21}$. The QD model is understandably favoured for lower wire diameters, however in light of our larger 100 nm diameter and the weak lateral conferment of the higher effective mass holes, we consider our heterostructures to be QWs. 

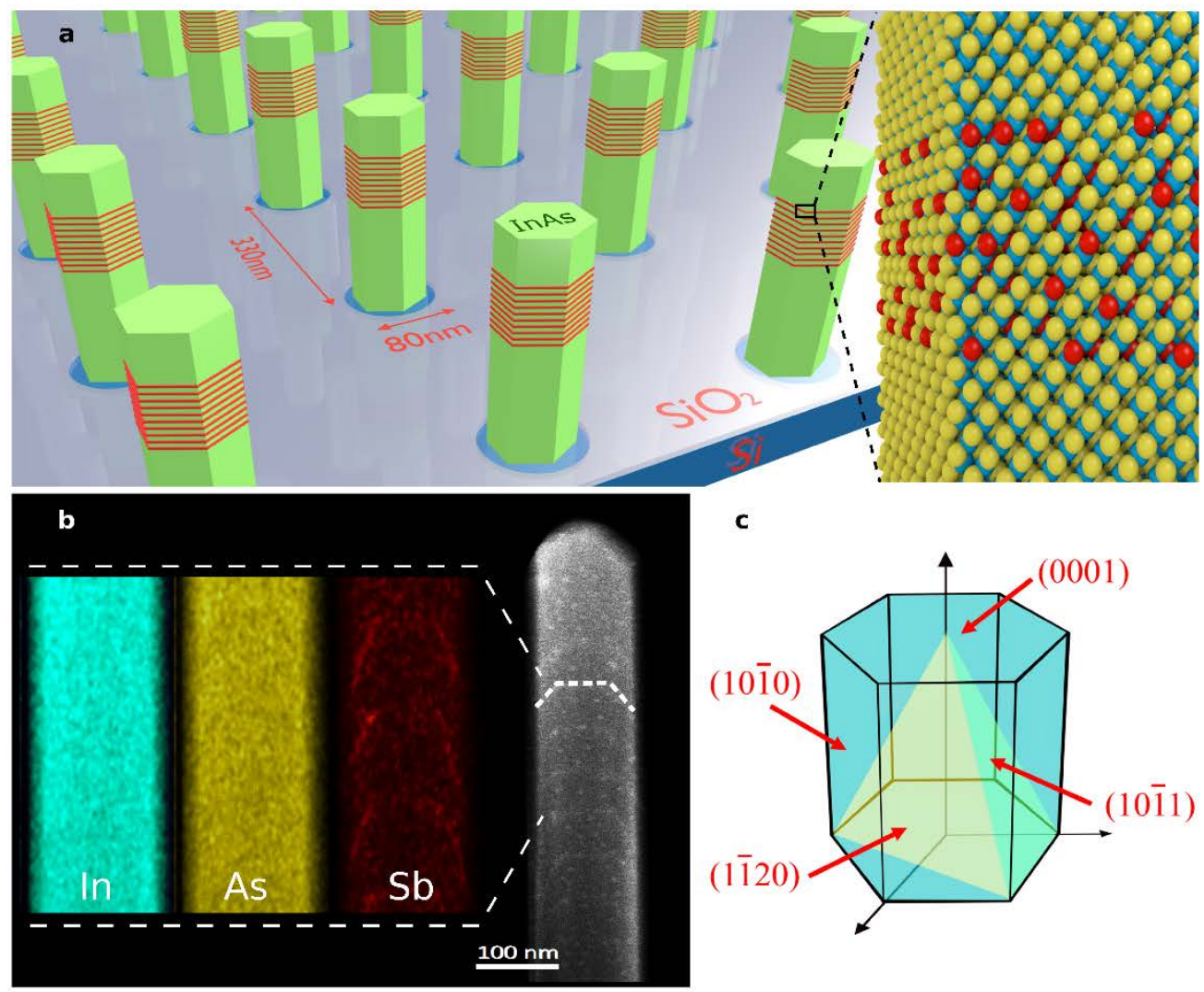

C

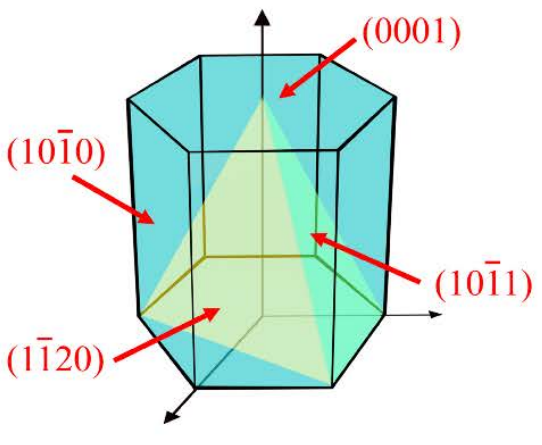

Figure 1 | Nanowire structural design and analysis. a, Schematic drawing of the InAsSb/InAs MQWs within InAs NWs, grown in a $330 \mathrm{~nm}$ pitch square array patterned on a silicon substrate using a $\mathrm{SiO}_{2}$ template with $80 \mathrm{~nm}$ diameter holes. Blue and yellow spheres represent In and As atoms respectively, while red spheres indicate the random incorporation of Sb within the quantum well region. b, STEM image showing the distribution of In, As and Sb obtained from 2D EDXS mapping and the resulting unusual facetted conical shape of the InAsSb MQWs. c, The unit cell of the WZ crystal structure showing the possible growth planes for the InAsSb MQW facets.

STEM analysis of the planes with preferential Sb incorporation show the angle to be consistent with $\{10 \overline{1} 1\}$ planes $\left(62^{\circ}\right)$, rather than the alternative $\{1 \overline{1} 20\}$ planes $\left(73^{\circ}\right)$, as shown in fig. $1(\mathrm{c})$. We reason that the InAs wire is faceted so that during InAsSb QW growth the Sb adatoms have a choice of incorporating on the $\{0001\}$ or $\{10 \overline{1} 1\}$ planes and conclude that bonding onto the $\{10 \overline{1} 1\}$ planes must be energetically favoured. Interestingly, close inspection of STEM images shows the InAsSb QWs maintain the WZ phase of the underlying InAs NW (supplementary info fig. S1). With extended growth durations, as employed in all earlier studies, preferential incorporation on the $\{10 \overline{1} 1\}$ planes would lead to a flat topped wire forming. This would then enable the $60^{\circ}$ rotation of the lattice around the 
[0001] axis, required for the transition to a ZB structure ${ }^{\text {Error! Bookmark not defined. }}$ In this work however, the QW growth durations are insufficient to allow a flat top to form and the WZ phase is uniquely maintained within the InAsSb.

The influence of the quantum wells on the optical emission from the NWs was studied using temperature dependent micro-PL spectroscopy. A comparison of the spectra measured at $4 \mathrm{~K}$ from the MQW, InAsSb, and InAs NWs is shown in fig. 2. Emission from the InAsSb NWs peaks at $0.380 \mathrm{eV}$ corresponding to $6 \%$ Sb with a dominant ZB phase in agreement with previous work ${ }^{9}$. A shoulder on the main peak can be observed originating from ZB InAs, which appears as the dominant phase in the very early stage of all our InAs NW growths ${ }^{9}$. Emission from the InAs NWs peaks at $0.482 \mathrm{eV}$, consistent with earlier work and a dominant WZ phase $\mathrm{e}^{22,23}$. 


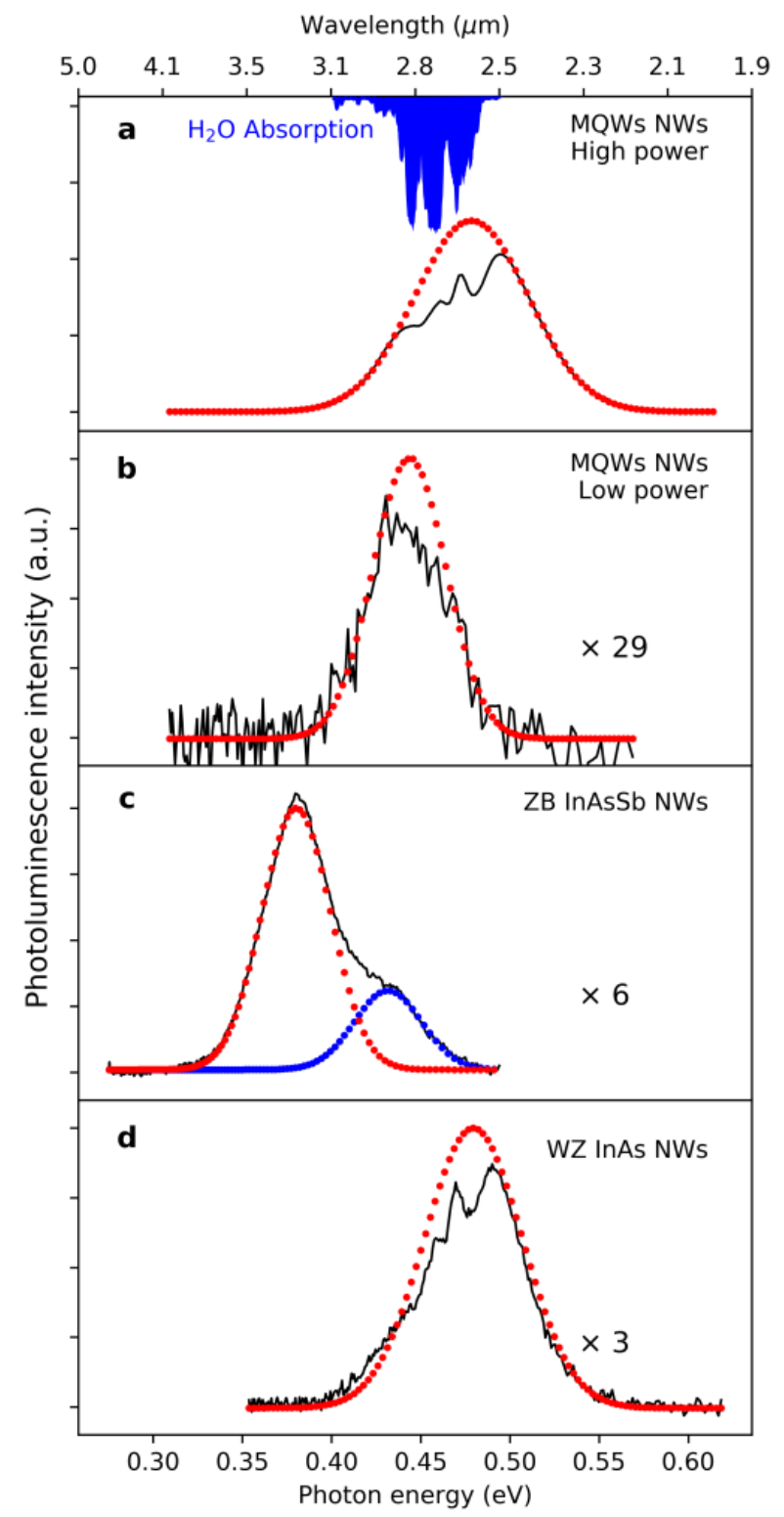

Figure 2 | Micro-PL spectra obtained at 4 K. The emission spectra measured from a, InAsSb/InAs MQW NWs at high power $\left(1.2 \times 10^{4} \mathrm{Wcm}^{-2}\right), \mathbf{b}, \mathrm{InAsSb} / \mathrm{InAs}$ MQW NWs at low power $\left(80 \mathrm{Wcm}^{-2}\right), \mathbf{c}$, InAsSb NWs at $\left(1.2 \times 10^{4} \mathrm{Wcm}^{-2}\right)$ and $\mathbf{d}$, InAs NWs at $\left(1.2 \times 10^{4} \mathrm{Wcm}^{-2}\right)$. The dotted lines represent Gaussian fits to the spectra, which also reveal the atmospheric absorption from water vapour $^{24}$ in this spectral region, as shown in a.

The spectra from the MQW NWs exhibit a clear increase in peak emission energy with respect to the InAsSb NWs, consistent with the strong carrier confinement within the quantum wells. The spectra are inhomogeneously broadened due to local growth rate driven length variations in the NWs, which also result in thickness variations of 
the QWs. Emission originates from type II recombination of electrons in the InAs layers with confined hole states in the InAsSb QWs, where the spatial separation helps to reduce non-radiative Auger recombination, with a corresponding increase in radiative emission ${ }^{25}$. Notably this results in an increased emission intensity at all temperatures. The emission intensity can be scaled to account for the reduced cross-sectional area of the nanowire samples and allow comparison with a bulk InAs reference, since only 7\% of the surface area is covered by the NWs even assuming a $100 \%$ nucleation yield in the mask sites. Accounting for this we observed that, under $3.2 \times 10^{4}$ Wcm ${ }^{-}$ ${ }^{2}$ excitation the MQW NW emission intensity is 4.2 fold enhanced with respect to a bulk InAs reference sample, compared to a 1.6 fold enhancement for the InAs NWs (supplementary information fig. S2).

The InAs NW emission is blue-shifted with respect to bulk InAs (ZB), due to the WZ crystal structure of the NW. Peak emission energy ranges from $0.473 \mathrm{eV}\left(1.1 \times 10^{3} \mathrm{Wcm}^{-2}\right.$ excitation, ) to $0.494 \mathrm{eV}\left(3.8 \times 10^{4} \mathrm{Wcm}^{-2} \mathrm{excitation}\right)$, consistent with earlier studies which reported bandgaps in the range $0.477 \mathrm{eV}$ to $0.540 \mathrm{eV}^{22,23,26}$. The PL emission energy from the InAs NWs blue shifted by $21 \mathrm{meV}$ over the range of pump powers used in our experiments, which is similar to that obtained by others ${ }^{26}$ and is associated with band filling. The commensurate shift for the MQW NWs is much larger, at 45 meV (supplementary information Fig. S4). The majority of this blue shift occurs at low pump powers, before the dependence becomes similar to that for pure InAs NWs at higher powers. The blue shift in the MQW NW emission arises due to band bending effects characteristic of type II QWs. The bending originates from Coulomb attraction between localised holes in the InAsSb QW attracting electrons from the adjacent InAs barrier, forming triangular quantum wells as shown in fig. 3(b). An increase in laser power, will raise the confining potential and consequently the electron quantization energy $\mathrm{E}$, with a typical $\Delta \mathrm{E} \sim \mathrm{L}^{1 / 3}$ behaviour where $\mathrm{L}$ is the integrated PL intensity ${ }^{27}$. The flat band transition energy within the InAsSb/InAs MQWs can thus be extracted from the $y$-axis intercept of fig. 3(a), as $0.438 \mathrm{eV}$. This is in good agreement with the expected transition energy we calculate, $0.443 \mathrm{eV}$, as shown in fig. 3(b). Full details of how the band diagram was developed and the transition energy calculated, are given in the supplementary information. 


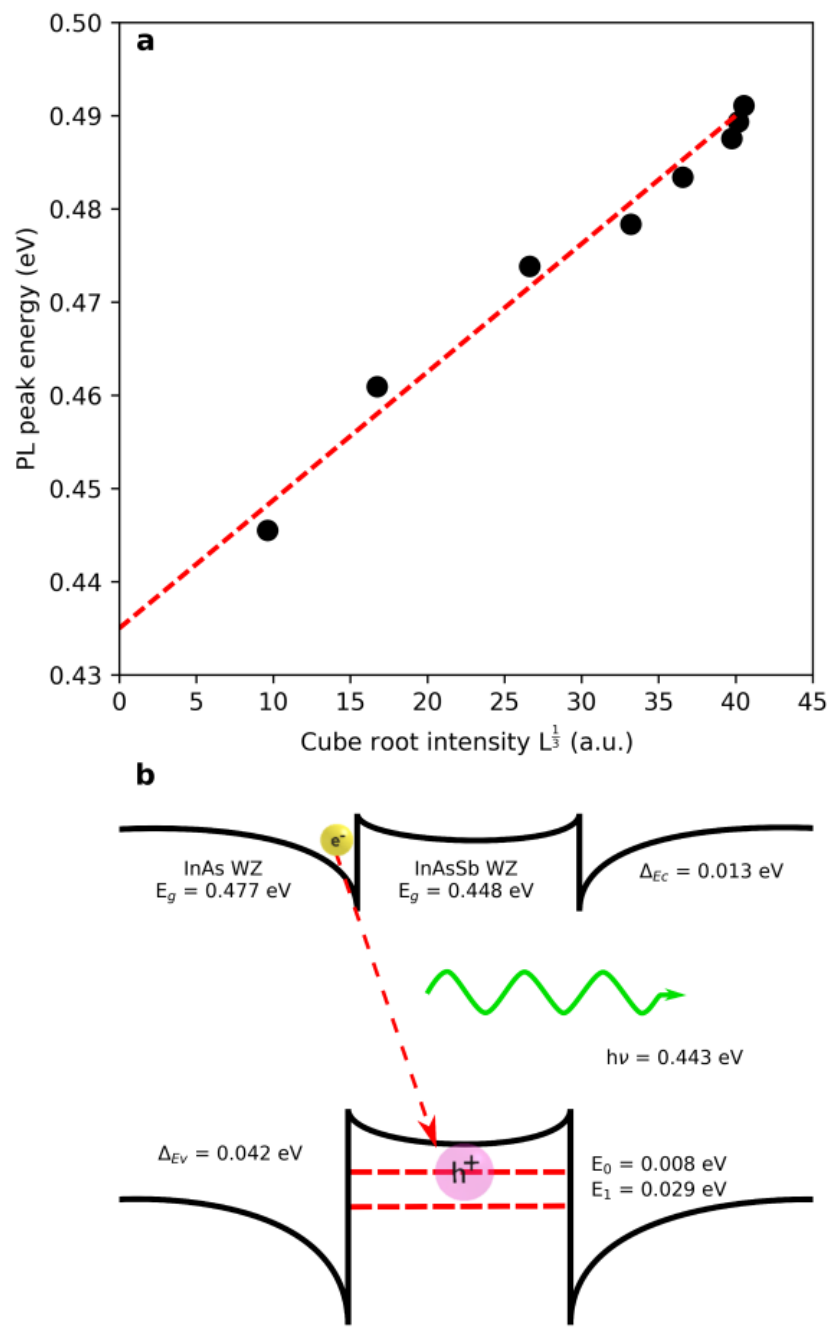

Figure 3 | Determination of the flat band transition energy at the QW. a, PL peak energy vs cube root of integrated PL intensity, elucidating the charging of the type II QW and allowing the flat band transition energy to be identified. b, The calculated band diagram for the InAsSb/InAs QW and photon energy, showing the band bending and triangular well formation.

Temperature dependent PL characterisation in fig. 4 shows that the MQW NWs exhibit strong PL emission persisting up to room temperature. This indicates that radiative recombination occurs primarily in the MQW away from the surface, which in InAs NWs is known to be accumulated due to Fermi level pinning, resulting in a low efficiency for radiative emission ${ }^{28}$. Hence, due to the quantum confinement achieved with the addition of the MQWs to the NWs, we obtain room temperature emission without passivation. Varshni relationships, fitted to this temperature dependent PL data, are given in the supplementary information (fig.S5). 


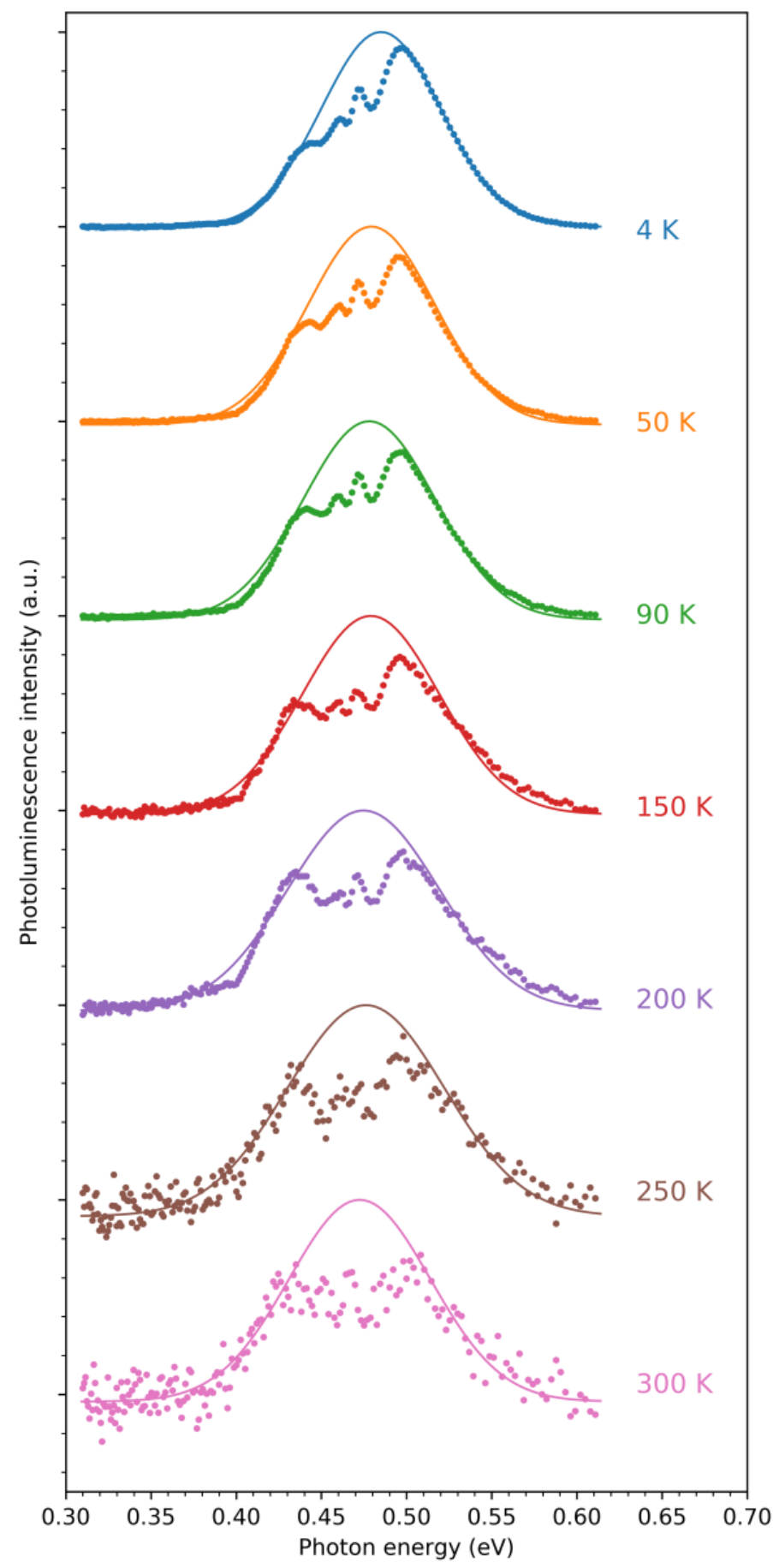

Figure 4 | Temperature dependence of the PL emission spectra obtained from the InAsSb MQW NWs. Emission spectra measured over the range 4-300 $\mathrm{K}$ using high excitation $\left(2.6 \times 10^{4} \mathrm{Wcm}^{-2}\right)$, showing the room temperature emission required for future practical NW infrared emitters. Dotted lines indicate Gaussian fits used to extract the peak emission wavelength. Atmospheric water vapour absorption is again evident in all the spectra. 
Figure 5(a) presents a comparison of the temperature quenching of the NW samples, where the superior performance of the MQW NWs is clearly evident due to the increase in the radiative emission rate and suppression of Auger recombination. An Arrhenius plot is shown in fig. 5(b) for the InAsSb MQW NWs from which an activation energy of $49 \mathrm{meV}$ was obtained from the high temperature region and $\sim 5 \mathrm{meV}$ for the low temperature region. This is approximately in agreement with the confinement energy for thermal excitation of holes out of the QW (hole localisation energy of $34 \mathrm{meV}$ ) and electrons from the interface triangular QWs ( $<13 \mathrm{meV}$ localisation energy) respectively.

The InAsSb MQW NWs exhibit increased emission intensity and superior temperature quenching behaviour compared with the bulk NWs as expected. Most notably the emission intensity is enhanced at all temperatures, due to the quantum confinement of electrons and holes. PL originates from type II recombination of electrons in the InAs layers with confined hole states in the InAsSb QWs, where the spatial separation helps to suppress Auger recombination. The activation energy of $\sim 49 \mathrm{meV}$ obtained from the Arrhenius plot is consistent with quenching due to carriers escaping confinement rather than Auger recombination and so provides indirect evidence for Auger suppression.

In type II QWs the Auger rate is determined by the overlap integral (between initial and final electron states at small transferred momentum) which is a minimum when the valence band offset is about three times the conduction band offset. Meanwhile, the radiative rate does not depend on the final state of any excited carrier, since it is a two body process, so the radiative rate can remain comparable with that in a type I QW ${ }^{29}$. 
a

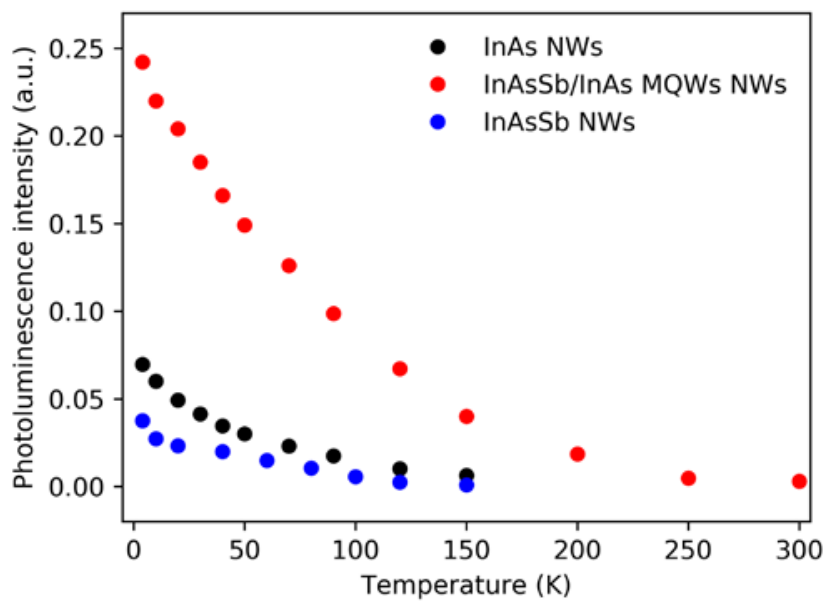

b

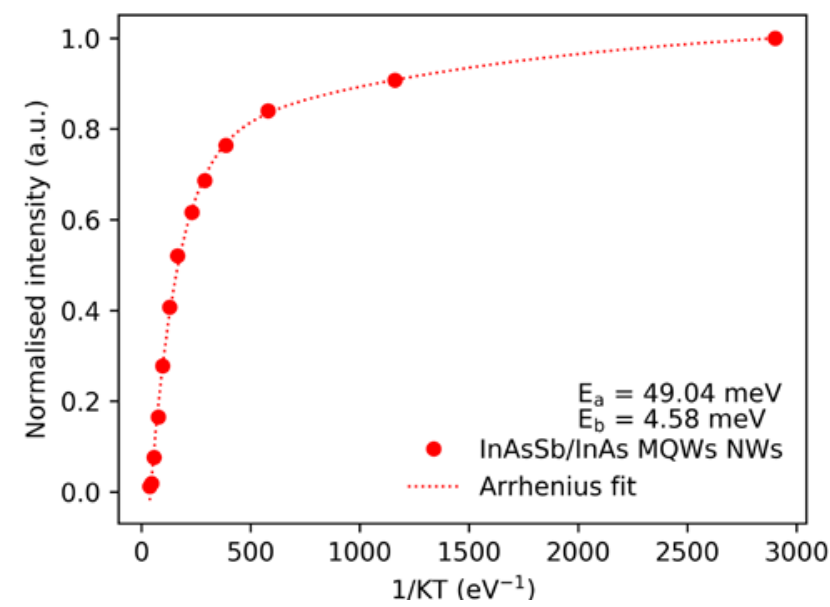

Figure 5 | Temperature dependence of the PL emission intensity from the InAsSb/InAs MQW NWs, InAsSb NWs and InAs NWs. a, The integrated PL intensities as a function of temperature, showing the quenching behaviour of the three NW samples. b, An Arrhenius plot of integrated PL intensity as a function of inverse temperature for the InAsSb/InAs MQW NWs, used to extract activation energies for the mechanisms driving thermal quenching.

In conclusion, we have demonstrated entirely new conical InAsSb MQWs in InAs NWs which exhibit mid-infrared emission up to room temperature. The nanowires were grown by catalyst-free selective area epitaxy on (111) Si substrates. Site-controlled growth allows precise geometric arrangement of the wires, which can be used to tune the optical properties and facilitates integration with lithographic processes and mature silicon electronics technology. This is a much-preferred fabrication route compared with conventional metal catalysts which introduce trap states within the underlying silicon and act as impurities within the NWs. STEM measurements revealed that these NWs exhibit an intriguing structure derived from the facets of the WZ crystal phase. This is in direct contrast to the 
growth of bulk InAsSb nanowires which are predominantly zinc-blende. These novel conical quantum wells confine the electron-hole recombination to the quantum well region, removing recombination via surface states which avoids the need for complex passivation schemes. Low-temperature micro-photoluminescence measurements revealed a clear shift in the emission energy with respect to the InAsSb NWs due to strong hole confinement which also increased the emission intensity by a factor of 4.2. A strong blue-shift was observed with increasing excitation power which is characteristic of type II QWs and is due to electron charging in triangular quantum wells formed at the interfaces. This substantially improves the electron-hole wavefunction overlap, increasing from approximately 40\% to 70\%, whilst providing Auger suppression resulting in enhanced PL emission which persists up to room temperature. The flat band transition energy was derived from the charging effect and calculated to be $0.438 \mathrm{eV}$, equivalent to a wavelength of $2.83 \mu \mathrm{m}$, in good agreement with calculations. These new quantum structured NWs will create a development pathway for novel nano-photonic and quantum light sources operating in the technologically important mid and far-infrared spectral ranges. They will be able to exploit the advantages of site controlled NWs including on-chip silicon integration and also enhance light-mater coupling based on their dimensions and geometry, opening the way for a wide range of applications.

\section{METHODS}

\section{Sample preparation and growth}

The InAs NWs were grown using self-assembled molecular beam epitaxy (SA-MBE) on $1 \times 10^{18} \mathrm{~cm}^{-3}$ boron doped ptype silicon (111) substrates, using a 25nm thick, thermally grown $\mathrm{SiO}_{2}$ template. Using positive e-beam resist AR-P 6200 (CSAR-62), arrays of $50 \mathrm{~nm}$ diameter holes were patterned in a $330 \mathrm{~nm}$ pitch square array using a JEOL JBX5500FS e-beam writer. The underlying $\mathrm{SiO}_{2}$ layer was etched using buffered hydrofluoric acid (BHF) solution, before the resist was removed using resist stripper with ultrasonic cleaning. Immediately before loading to the MBE reactor, the sample was given an additional, unmasked, dip in BHF. This ensures the presence of an oxide free, hydrogen terminated silicon surface within the hole. The final hole diameter was $\sim 80 \mathrm{~nm}$ due to lateral etching. Growth was carried out using a solid source VG V80h MBE reactor and 2D layer growth rates of $0.08 \mathrm{ML} / \mathrm{s}$ for In, $1.59 \mathrm{ML} / \mathrm{s}$ for As and 0.36 ML/s for Sb were employed. These growth rates were calibrated for 2D ZB growth on 
(001) InAs, GaAs and InSb substrates respectively. The substrates were annealed in the growth chamber at $600^{\circ} \mathrm{C}$ for 10 minutes to remove any contamination and then cooled to the growth temperature of $480^{\circ} \mathrm{C}$. The $\mathrm{MQW}$ NW growth was initiated by opening the As shutter, followed by the In shutter 20 seconds later. Following the growth of an initial pure InAs section for 1 hour, the MQW active region was grown as 10 repeats of $\operatorname{InAs}_{1-\mathrm{x}} \mathrm{Sb}_{\mathrm{x}} / \mathrm{InAs}_{\mathrm{MQW}}$ with growth durations of 180s and 27s respectively. This was expected to form $25 \mathrm{~nm}$ thick InAs barriers and $8 \mathrm{~nm}$ InAsSb wells, with a total active region thickness of $330 \mathrm{~nm}$. Finally, the wires were finished with an InAs cap, grown for 10 minutes. The wires were on average $1.5 \mu \mathrm{m}$ in length and $100 \mathrm{~nm}$ in diameter (see supplementary information S6). The same fluxes and temperatures were used during the growth of the pure InAs and pure InAsSb NWs. A planar bulk InAs sample was also grown on an InAs substrate, as a further reference.

\section{Nano-structural analysis}

The morphology of the wires, including the diameter and height was studied using an Annular dark field (ADF) scanning transmission electron microscopy (STEM), images were taken in an aberration-corrected JEOL ARM200F microscope working at $200 \mathrm{kV}$.

\section{Photoluminescence spectroscopy}

For micro-PL experiments, the samples were placed in an Oxford Instruments continuous flow He cryostat which enabled temperature selection in the range 4-300 K. A confocal optical configuration was employed using an 808 nm laser with excitation powers up to $476 \mathrm{~mW}$. The emitted photoluminescence was collected using a Bentham 0.3 m monochromator and collected by a liquid $\mathrm{N}_{2}$ cooled InSb photodetector. The excitation laser spot was focused onto the wires with a spot diameter of $\sim 40 \mu \mathrm{m}$ giving a power density around $10^{4} \mathrm{~W} / \mathrm{cm}^{2}$. 


\section{ASSOCIATED CONTENT}

Supporting Information

High resolution TEM structural analysis of the quantum wells, , SEM images of grown NWs and further PL analysis including: PL intensity comparison at 4K, temperature dependent PL emission from InAs and InAsSb NWs, dependence of PL emission energy with pump power, temperature dependence of PL emission with Varshni parameters and development of band structure for the MQWs.

\section{ACKNOWEDGEMENTS}

The authors would like to thank Mark C. Rosamond and Edmund H. Linfield from the University of Leeds for their help with proximity effect correcting the e-beam masks.

The authors gratefully acknowledge funding support for this work from the UK Centre for Defence Enterprise (Grant No. CDE65947). A.A has been supported by a studentship funded by the Qassim University in Saudi Arabia. A.R.J.M and P.J.C have been supported by individual research fellowships from the Royal Academy of Engineering.

\section{AUTHOR CONTRIBUTIONS}

A.A., A.K. and A.R.J.M. conceived the project. A.A., M.D.T. and A.R.J.M, designed the arrays, and performed the NW growth. Y.N and R.J.Y developed and performed patterning of the growth templates. A.M.S. provided TEM analysis. A.A, P.J.C. and A.K. performed and interpreted the photoluminescence. A.A, P.J.C, A.K. and A.R.J.M. cowrote the paper.

\section{COMPETING FINANCIAL INTERESTS}

The authors declare no competing financial interests. 


\section{REFERENCES}

\footnotetext{
1 Joyce, H.; Gao, Q.; Hoe Tan, H.; Jagadish, C.; Kim, Y.; Zou, J.; Smith, L.; Jackson, H.; Yarrison-Rice, J.; Parkinson, P. et al. III-V Semiconductor Nanowires for Optoelectronic Device Applications. Progress in Quantum Electronics 2011, 35, 23-75.
}

${ }^{2}$ Thelander, C.; Björk, M.; Larsson, M.; Hansen, A.; Wallenberg, L.; Samuelson, L. Electron Transport in InAs Nanowires and Heterostructure Nanowire Devices. Solid State Communications 2004, 131, 573-579.

${ }^{3}$ Aharonovich, I.; Englund, D.; Toth, M. Solid-State Single-Photon Emitters. Nat. Photon. 2016, 10, $631-641$.

${ }^{4}$ Dayeh, S.; Aplin, D.; Zhou, X.; Yu, P.; Yu, E.; Wang, D. High Electron Mobility InAs Nanowire Field-Effect Transistors. Small 2007, 3, 326-332.

5 Svensson, J.; Anttu, N.; Vainorius, N.; Borg, B.; Wernersson, L. Diameter-Dependent Photocurrent in InAsSb Nanowire Infrared Photodetectors. Nano Lett 2013, 13, 1380-1385.

${ }^{6}$ Scofield, A.; Kim, S.; Shapiro, J.; Lin, A.; Liang, B.; Scherer, A.; Huffaker, D. Bottom-up Photonic Crystal Lasers. Nano Lett. 2011, 11, 5387-5390.

${ }^{7}$ Kim, H., Farrell, A., Senanayake, P., Lee, W. and Huffaker, D. Monolithically Integrated InGaAs Nanowires on 3D Structured Silicon-on-Insulator as a New Platform for Full Optical Links. Nano Lett. 2016, 16, $1833-1839$.

${ }^{8}$ Miao, J.; Hu, W.; Guo, N.; Lu, Z.; Zou, X.; Liao, L.; Shi, S.; Chen, P.; Fan, Z.; Ho, J. et al. Single InAs Nanowire Room-Temperature Near-Infrared Photodetectors. ACS Nano 2014, 8, 3628-3635.

9 Thompson, M.; Alhodaib, A.; Craig, A.; Robson, A.; Aziz, A.; Krier, A.; Svensson, J.; Wernersson, L.; Sanchez, A.; Marshall, A. Low Leakage-Current InAsSb Nanowire Photodetectors on Silicon. Nano Lett 2016, 16, $182-187$.

10 Potts, H.; Friedl, M.; Amaduzzi, F.; Tang, K.; Tütüncüoglu, G.; Matteini, F.; Alarcon Lladó, E.; McIntyre, P.; Fontcuberta i Morral, A. From Twinning to Pure Zincblende Catalyst-Free InAs(Sb) Nanowires. Nano Lett. 2016, 16, 637-643.

${ }^{11}$ Sun, M.; Leong, E.; Chin, A.; Ning, C.; Cirlin, G.; Samsonenko, Y.; Dubrovskii, V.; Chuang, L.; Chang-Hasnain, C. Photoluminescence Properties of InAs Nanowires Grown on GaAs and Si Substrates. Nanotechnology 2010, 21, 335705.

${ }^{12}$ Koblmüller, G.; Vizbaras, K.; Hertenberger, S.; Bolte, S.; Rudolph, D.; Becker, J.; Döblinger, M.; Amann, M.; Finley, J.; Abstreiter, G. Diameter Dependent Optical Emission Properties of InAs Nanowires Grown on Si. Appl. Phys. Lett. 2012, 101, 053103.

${ }^{13}$ Farrell, A.; Lee, W.; Senanayake, P.; Haddad, M.; Prikhodko, S.; Huffaker, D. High-Quality InAsSb Nanowires Grown by Catalyst-Free Selective-Area Metal-Organic Chemical Vapor Deposition. Nano Lett. 2015, 15, 66146619.

${ }^{14}$ Treu, J.; Bormann, M.; Schmeiduch, H.; Döblinger, M.; Morkötter, S.; Matich, S.; Wiecha, P.; Saller, K.; Mayer, B.; Bichler, M. et al. Enhanced Luminescence Properties of InAs-InAP Core-Shell Nanowires. Nano Lett. 2013, 13, 6070-6077.

${ }^{15}$ Hjort, M.; Lehmann, S.; Knutsson, J.; Zakharov, A.; Du, Y.; Sakong, S.; Timm, R.; Nylund, G.; Lundgren, E.; Kratzer, P. et al. Electronic and Structural Differences Between Wurtzite and Zinc Blende InAs Nanowire Surfaces: Experiment and Theory. ACS Nano 2014, 8, 12346-12355.

16 Sourribes, M.; Isakov, I.; Panfilova, M.; Liu, H.; Warburton, P. Mobility Enhancement by Sb-Mediated Minimisation of Stacking Fault Density in InAs Nanowires Grown on Silicon. Nano Lett. 2014, 14, $1643-1650$. 
${ }^{17}$ Wu, J.; Ramsay, A.; Sanchez, A.; Zhang, Y.; Kim, D.; Brossard, F.; Hu, X.; Benamara, M.; Ware, M.; Mazur, Y. et al. Defect-Free Self-Catalyzed GaAs/GaAsP Nanowire Quantum Dots Grown on Silicon Substrate. Nano Letters 2015, 16, 504-511.

${ }^{18}$ Reimer, M.; Bulgarini, G.; Akopian, N.; Hocevar, M.; Bavinck, M.; Verheijen, M.; Bakkers, E.; Kouwenhoven, L.; Zwiller, V. Bright Single-Photon Sources in Bottom-up Tailored Nanowires. Nature Communications 2012, 3, 737.

${ }^{19}$ Yang, L.; Motohisa, J.; Takeda, J.; Tomioka, K.; Fukui, T. Size-Dependent Photoluminescence of Hexagonal Nanopillars With Single InGaAs/GaAs Quantum Wells Fabricated by Selective-Area Metal Organic Vapor Phase Epitaxy. Applied Physics Letters 2006, 89, 203110.

${ }^{20}$ Ra, Y.; Navamathavan, R.; Park, J.; Lee, C. High-Quality Uniaxial $\operatorname{In}_{\mathrm{x}} \mathrm{Ga}_{1-\mathrm{X}} \mathrm{N} / \mathrm{GaN}$ Multiple Quantum Well (MQW) Nanowires (NWs) on Si (111) Grown by Metal-Organic Chemical Vapor Deposition (MOCVD) and LightEmitting Diode (LED) Fabrication. ACS Applied Materials \& Interfaces 2013, 5, 2111-2117.

21 Armitage, R.; Tsubaki, K. Multicolour Luminescence from InGaN Quantum Wells Grown Over GaN Nanowire Arrays by Molecular-Beam Epitaxy. Nanotechnology 2010, 21, 195202.

${ }^{22}$ Rota, M.; Ameruddin, A.; Fonseka, H.; Gao, Q.; Mura, F.; Polimeni, A.; Miriametro, A.; Tan, H.; Jagadish, C.; Capizzi, M. Bandgap Energy of Wurtzite InAs Nanowires. Nano Lett. 2016, 16, 5197-5203.

${ }^{23}$ Dick, K.; Thelander, C.; Samuelson, L.; Caroff, P. Crystal Phase Engineering in Single InAs Nanowires. Nano Lett. 2010, 10, 3494-3499.

${ }^{24}$ Lord, S.D., NASA Tech. Memo. 1992,103957.

25 Carrington, P.; Zhuang, Q.; Yin, M.; Krier, A. Temperature Dependence of Mid-Infrared Electroluminescence in Type II InAsSb/InAs Multi-Quantum Well Light-Emitting Diodes. Semicond. Sci. Technol. 2009, $24,075001$.

${ }^{26}$ Möller, M.; de Lima Jr, M.; Cantarero, A.; Chiaramonte, T.; Cotta, M.; Iikawa, F. Optical Emission of InAs Nanowires. Nanotechnology 2012, 23, 375704.

${ }^{27}$ Ledentsov, N.; Böhrer, J.; Beer, M.; Heinrichsdorff, F.; Grundmann, M.; Bimberg, D.; Ivanov, S.; Meltser, B.; Shaposhnikov, S.; Yassievich, I. et al. Radiative States in Type-II GaSb/GaAs Quantum Wells. Phys. Rev. B 1995, 52, 14058-14066.

${ }^{28}$ Olsson, L. Ö.; M. Andersson, C. B.; Håkansson, M. C.; Kanski, J.; Ilver, L.; and Karlsson U. O.;Charge Accumulation at InAs Surfaces. Phys. Rev. Lett. 1996, 76, 3626-3629.

29 Zegrya G.G. and Andreev A.D. Mechanism of suppression of Auger recombination processes in type II heterostructures, Appl. Phys. Lett. 1995, 67(18) 2681. 


\section{FIGURE LEGENDS}

Figure 1 | Nanowire structural design and analysis. a, Schematic drawing of the InAsSb/InAs MQWs within InAs NWs, grown in a $330 \mathrm{~nm}$ pitch square array patterned on a silicon substrate using a $\mathrm{SiO}_{2}$ template with $80 \mathrm{~nm}$ diameter holes. Blue and yellow spheres represent In and As atoms respectively, while red spheres indicate the random incorporation of Sb within the quantum well region. b, STEM image showing the distribution of In, As and the Sb obtained from 2D EDXS mapping and the resulting unusual facetted conical shape of the InAsSb MQWs. c, The unit cell of the WZ crystal structure showing the possible growth planes for the InAsSb MQW facets.

Figure 2 | Micro-PL spectra obtained at 4 K. The emission spectra measured from a, InAsSb/InAs MQW NWs at high power $\left(1.2 \times 10^{4} \mathrm{Wcm}^{-2}\right), \mathbf{b}, \mathrm{InAsSb} / \mathrm{InAs}$ MQW NWs at low power $\left(80 \mathrm{Wcm}^{-2}\right), \mathbf{c}$, InAsSb NWs at $\left(1.2 \times 10^{4}\right.$ $\left.\mathrm{Wcm}^{-2}\right)$ and $\mathbf{d}$, InAs NWs at $\left(1.2 \times 10^{4} \mathrm{Wcm}^{-2}\right)$. The dotted lines represent Gaussian fits to the spectra, which also reveal the atmospheric absorption from water vapour ${ }^{19}$ in this spectral region, as shown in a.

Figure 3 | Determination of the flat band transition energy at the QW. a, PL peak energy vs cube root of integrated PL intensity, elucidating the charging of the type II QW and allowing the flat band transition energy to be identified. b, The calculated band diagram for the InAsSb/InAs QW and photon energy, showing the band bending and triangular well formation.

Figure 4 | Temperature dependence of the PL emission spectra obtained from the InAsSb MQW NWs. Emission spectra measured over the range 4-300 $\mathrm{K}$ using high excitation $\left(2.6 \times 10^{4} \mathrm{Wcm}^{-2}\right)$, showing the room temperature emission required for future practical NW infrared emitters. Dotted lines indicate Gaussian fits used to extract the peak emission wavelength. Atmospheric water vapour absorption is again evident in all the spectra.

Figure 5 | Temperature dependence of the PL emission intensity from the InAsSb/InAs MQW NWs, InAsSb NWs and InAs NWs. a, The integrated PL intensities as a function of temperature, showing the quenching behaviour of the three NW 
samples. b, An Arrhenius plot of integrated PL intensity as a function of inverse temperature for the InAsSb/InAs MQW NWs, used to extract activation energies for the mechanisms driving thermal quenching.

\section{The TOC figure}

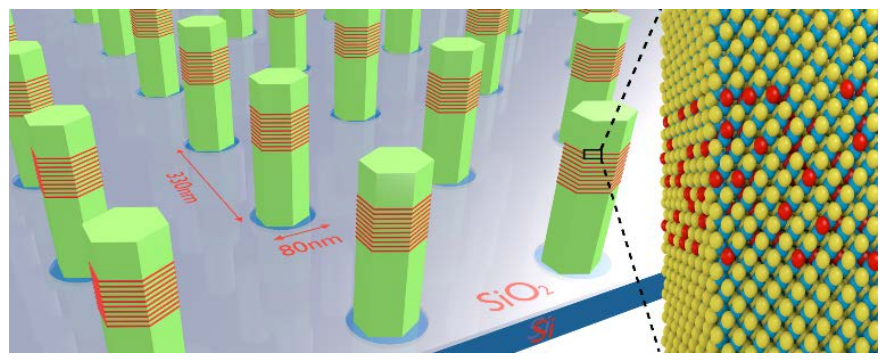

